etry yields a value $|(1 / n) /(d n / d z)|^{-1}=L=2 \pm 1 \mathrm{~mm}$ at $r=0$. It may be shown that at resonance

$$
\Delta \omega=\frac{-\omega_{0}}{2}\left(1-\frac{9}{4} \frac{T_{e}}{m_{e} c^{2}}-\frac{9}{2} \frac{\Delta \overrightarrow{\mathrm{k}} \cdot \hat{z}}{k_{0}} \frac{T_{e}}{m_{e} c^{2}}\right),
$$

and that at any position $(\Delta \omega)^{2}=\omega_{p e}{ }^{2}+3 \Delta k^{2} \boldsymbol{T}_{e} / m_{e}$. (The other sideband has $\omega^{\prime}=\omega_{0}+\Delta \omega_{0}$ ) From Eq. (4) it can be shown that if we depart a distance $\Delta z$ from resonance, the plasma wave detected at this $\Delta z$ is at resonance at distance $\Delta z^{\prime}$ $\approx\left(2 \Delta \overrightarrow{\mathrm{k}} \cdot \hat{z} / k_{0}\right) \Delta z$ away. $\Delta z^{\prime}$ is then just the width of the convective instability, which for our experimental parameters may be numerically calculated to be $30 \pm 10 \mu \mathrm{m}$. Using these values, we may calculate $I$; matching $\ln I$ to the exponent in Eq. (3) and letting the pump power be $10^{11} \mathrm{~W} /$ $\mathrm{cm}^{2}$, we obtain $L=2.7 \mathrm{~mm}$, in qualitative agreement with the experimentally determined value.

In conclusion, we have observed the parametric decay of $\mathrm{CO}_{2}$-laser light into plasma waves at quarter-critical density; the amplitude and experimental parameters allow us to infer that this decay is convective. The technique of ruby-laser Thomson scattering in a $\mathrm{CO}_{2}$-laser-heated gastarget plasma seems to hold great promise as a tool for diagnosing parametric turbulence of interest to laser-fusion schemes.

The authors are happy to achnowledge their valuable discussions with Dr. E. Valeo concern- ing this work.

This work was supported by the U. S. Energy Research and Development Administration, Contract No. EY/76-C-02-3073.

\footnotetext{
(a) Present address: Massachusetts Institute of Technology, Cambridge, Mass。02139。

${ }^{1} \mathrm{~A}$ 。A. Offenberger, M. R。 Cervenan, A. M。 Yam, and A.W. Pasternak, J. Appl. Phys.47, 1451 (1976)。

${ }^{2}$ John J. Turechek and Francis F. Chen, Phys。Rev。 Lett. $\underline{36}, 720$ (1976).

${ }^{3}$ B. Grek, H. Pépin, and F. Rheault, Phys. Rev. Lett. 38, 898 (1977).

${ }^{4}$ Steven Jackel, Bruce Perry, and Moshe Lubin, Phys. Rev. Lett。37, 95 (1976).

${ }^{5}$ Steven Jackel, James Albritton, and Edward Goldman, Phys。Rev。Lett。35, 514 (1975)。

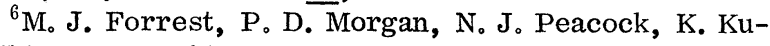
riki, M。 V. Goldman, and $T_{0}$ Rudolph, Phys。Rev. Lett. 37, 1681 (1976)。

${ }^{7}$ T。 K. Chu and L。C。Johnson, Phys。Fluids $\underline{18}, 1460$ (1975).

${ }^{8} \mathrm{E}$. Atlee Jackson, Phys. Rev. 153, 235 (1967)。

${ }^{9} \mathrm{~L}$. C. Johnson and T。K. Chu, Phys。Rev. Lett。 32 , 517 (1974).

${ }^{10}$ Optical fibers supplied by Bell Laboratories.

${ }^{11}$ Marshall N. Rosenbluth, Phys。Rev。Lett. $\underline{29}, 565$ (1972)。

${ }^{12}$ C. S。 Liu, Marshall N. Rosenbluth, and Roscoe B。 White, Phys. Fluids 17, 1211 (1974).

${ }^{13}$ C.S. Liu and Marshall No Rosenbluth, Phys. Fluids 19, 967 (1976).
}

\title{
Electromagnetic Structure Resonances in Inhomogeneous Plasma Layers
}

\author{
F. J. Mayer, R. K. Osborn, D. W. Daniels, and J. F. McGrath \\ KMS Fusion, Ann Arbor, Michigan
}

(Received 21 September 1977)

\begin{abstract}
Laser-light absorption and radiation forces are investigated for weakly collisional $(\nu / \omega<<1)$, rigid, two-step plasma profiles in planar and spherical geometry. For the first step near critical density, the second step above critical, and appropriate step lengths, we find strong absorption even for weakly collisional plasmas. We show, however, that the radiation forces may not permit such profiles to persist in realistic, dynamic situations.
\end{abstract}

The subject of electromagnetic-wave absorption in high-density plasmas has received considerable attention recently ${ }^{1,2}$ because of its importance to the laser-driven-fusion program. It is well known that the electron density profile which is produced not only strongly affects the relative importance of various absorption mechanisms, but also affects the strength and direction of the ponderomotive force $\mathrm{e}^{3.4}$ exerted on the ions through electrostatic coupling with the elec- trons. The ponderomotive force has also received considerable attention recently ${ }^{5-7}$ in regard to self-consistent density profile formation; not all treatments, however, account for absorption and some are not rigorous. In this Letter, we examine the transverse electromagnetic wave interaction for two very simple inhomogeneous plasma layers, a two-step planar plasma layer and a two-step spherical layer. In these cases, the plasma is forced to be rigid, i.e., no plasma 
motion is allowed, although there are unbalanced forces on the layers. We will exhibit the electromagnetic wave solutions explicitly, examine the "structure" resonances which arise due to coherence effects, and compute the ponderomotive forces and collisional absorption in resonant and nonresonant cases.

These two-step problems ${ }^{8}$ (at least in some respects) are essentially textbook exercises in coherent plasma optics. However, the implications of such a two-step structure for strong absorption and ponderomotive forces due to coherence in weakly collisional plasmas do not appear to have been explored in the laser-fusion context. A brief sketch of some results for the reflectivity of a two-step planar plasma has been presented, ${ }^{2}$ but appears to be somewhat discrepant with the results presented here. The linear-ramp problem has been studied ${ }^{4,8}$ to varying degrees; however, this profile does not, as strongly, exhibit the resonance effects.

Consider the two-step electron density profiles of Fig. 1. With the "cold" collisional plasma approximation, we have $\vec{J}=\sigma \vec{E}$ where the complex

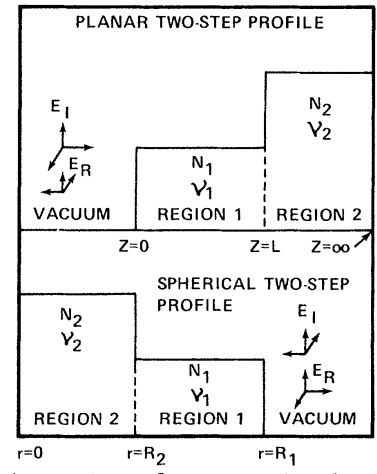

FIG. 1. The two-step planar and spherical electron density profiles. In the planar case the electromagnetic wave is incident from the left; in the spherical case the electromagnetic wave is incident from the right.

$\sigma$ is given by

$$
\sigma=(\omega / 4 \pi)\left(\omega_{p} / \omega\right)^{2}(\nu / \omega-i)^{-1},
$$

where $\omega_{p}$ is the (local) plasma frequency, and $\nu$ and $\omega$ are the (local) effective collision frequency and the wave frequency, respectively.

In the planar case, with $E$ along $x$, and $B$ along $y$, the solutions to Maxwell's equations in the various regions are given as follows:

incident: $E_{v}=\exp \left(i k_{0} z\right), B_{v}=\exp \left(i k_{0} z\right)$;

reflected: $E_{R}=R \exp \left(-i k_{0} z\right), B_{R}=-R \exp \left(-i k_{0} z\right)$;

region 1: $E_{1}=A \exp \left(i k_{1} z\right)+D \exp \left(-i k_{1} z\right), B_{1}=k_{1} k_{0}^{-1}\left[A \exp \left(i k_{1} z\right)-D \exp \left(-i k_{1} z\right)\right]$;

region 2: $E_{2}=C \exp \left(i k_{2} z\right), B_{2}=C k_{2} k_{0}{ }^{-1} \exp \left(i k_{2} z\right)$.

The incident field has unit amplitude ( $E_{0}$ in the figures), and there is no reflected wave in region 2. The $k$ 's are the complex wave numbers appropriate to each region, i.e.,

$$
k_{p}^{2}=k_{0}^{2}(1+4 \pi i \sigma / \omega), \quad k_{0}=\omega / c \text {. }
$$

Since there are no currents or free charges at the boundaries, the electric and magnetic fields must be continuous at $z=0, z=L$. Matching at the boundaries determines the unknown coefficients; they are given by

$$
\begin{aligned}
& R=\left[\left(k_{0}+k_{1}\right)\left(k_{1}-k_{2}\right) \exp \left(i k_{1} L\right)+\left(k_{0}-k_{1}\right)\left(k_{1}+k_{2}\right) \exp \left(-i k_{1} L\right)\right] / Q ; \\
& A=\left[2 k_{0}\left(k_{1}+k_{2}\right) \exp \left(-i k_{1} L\right)\right] / Q ; \quad D=\left[2 k_{0}\left(k_{1}-k_{2}\right) \exp \left(-i k_{1} L\right)\right] / Q ; \\
& C=\left[4 k_{0} k_{1} \exp \left(-i k_{2} L\right)\right] / Q ; \quad Q=\left(k_{0}-k_{1}\right)\left(k_{1}-k_{2}\right) \exp \left(i k_{1} L\right)+\left(k_{0}+k_{1}\right)\left(k_{1}+k_{2}\right) \exp \left(-i k_{1} L\right) .
\end{aligned}
$$

With these coefficients and the fields [Eq. (1)], all electromagnetic quantities are determined for an arbitrary two-step profile (note that a subset of the spatial structures considered here is the "square" slab"). Of particular interest is the power reflectivity, $R R^{*}$. Figure 2 shows $R R^{*}$ versus the length of the first plasma step in units of $k_{0} L$. The interesting coherence effects are manifested when the second step is overdense, i.e., $\left(\omega_{p} / \omega\right)^{2}>1$, so as to reflect the electromag- netic wave, and the first step is slightly underdense, $\left(\omega_{p} / \omega\right)^{2} \lesssim 1$. The four reflectivity curves correspond to increasing first-step densities and a low relative collision frequency of $\nu / \omega=0.001$ (for a Nd:glass laser plasma at $\sim 2 \mathrm{keV}$ ). As can be seen, the absorption $\left(1-R R^{*}\right)$ is quite low for low first-step density but exhibits strong absorption resonances when the first-step density approaches 1. The second-step density and colli- 


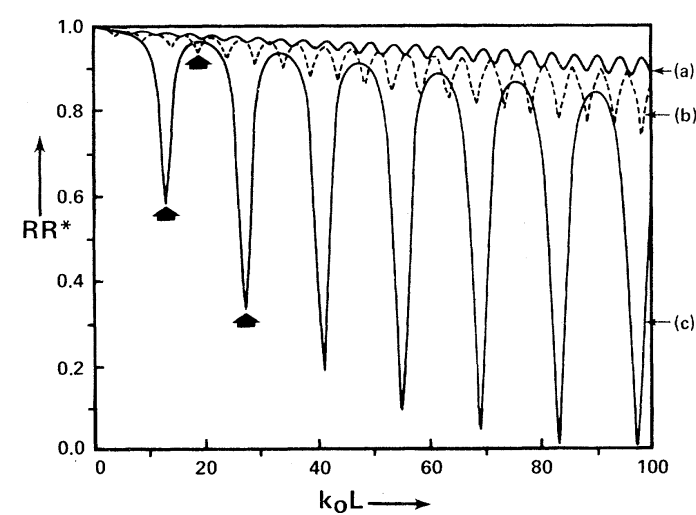

FIG. 2. The power reflectivity $R R^{*}$ vs $k_{0} L$ for the following parameters: $n_{2} / n_{c}=2.0 ; \nu_{2} / \omega=0.002 ; \nu_{1} / \omega$ $=0.001$. Curve $a, n_{1} / n_{c}=0.4$; curve $b, n_{1} / n_{c}=0.6$; curve $c, n_{1} / n_{c}=0.95$.

sion frequency have little effect except to shift the resonances slightly as long as the second step is overdense.

The strong absorption resonances obtained are due to the slowing of the electromagnetic wave in the first step, and to the matching of the plasma wavelength in the first step to the step size. Figure 3 shows the electric and magnetic energy densities for the first two "on-resonance" and first "off-resonance" cases corresponding to the arrows in Fig. 2. As is seen, the resonance cases have matched the step size and plasma wavelength, and produced a strongly increased electric field amplitude. The increased electric field in the first step gives rise to the increased collisional absorption since the Ohmic heating $\frac{1}{2} \operatorname{Re}(\overrightarrow{\mathrm{J}} \cdot \overrightarrow{\mathrm{E}} *)$ becomes large. Importantly, these structure resonances can show strong absorption even for a weakly collisional plasma.

Given the fields in the various regions, we may calculate the ponderomotive force on the plasma layer. The ponderomotive force (the time-averaged Lorentz force) is given by

$$
\overrightarrow{\mathrm{F}}_{p}=\operatorname{Re}(\overrightarrow{\mathrm{J}} \times \overrightarrow{\mathrm{B}} *) / 2 c .
$$

Figure 4 shows $F_{p}$ for the same two "on-resonance" and one "off-resonance" cases as in Fig. 3. Notice that the force at the very edge of the first step on the "on-resonance" case is directed toward the vacuum, and for the "off-resonance" case, the force is into the plasma. This situation holds generally; therefore the "on-resonance" cases which are more strongly absorbing will probably tend to be destabilizing, whereas the weakly absorbing "off-resonance" case will tend
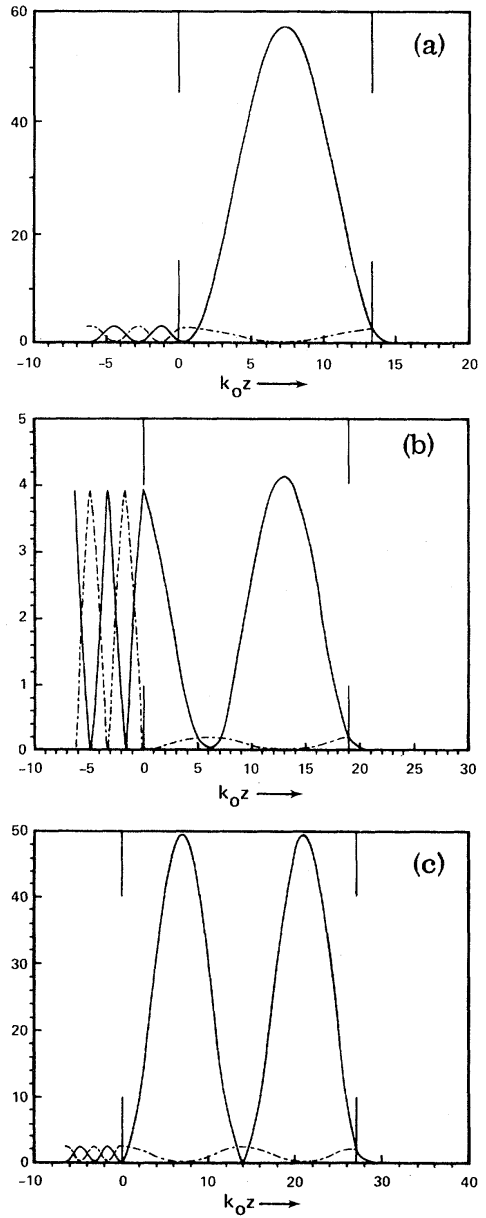

FIG. 3. The electric and magnetic field intensities, $E E^{*}$ and $B B^{*}$ (in units of $E_{0}{ }^{2}$ ), for (a), (c) the first two "on-resonance" cases and (b) the first "off-resonance" case indicated by the arrows in Fig. 2. $E E^{*}$, : $B B^{*},-\cdots$.

to be stabilizing. Notice also that the peak value of the ponderomotive force is more than an order of magnitude larger in the "on-resonance" cases than in the "off-resonance" cases. Of course, the real effects of hydrodynamic forces and flow, along with the fully consistent treatment of the electromagnetic wave, must be examined before the occurrence, let alone the stability, of such structures can be understood.

The spherical two-step plasma layer can be calculated in a manner similar to the planar problem, if we may employ the scalar (rather than vector) wave equations ${ }^{5}$ to describe the electromagnetic fields. The results are similar to the planar problem, except that there is no clear distinction between transmission and reflection. Figure 5 shows the power reflectivity for a few 

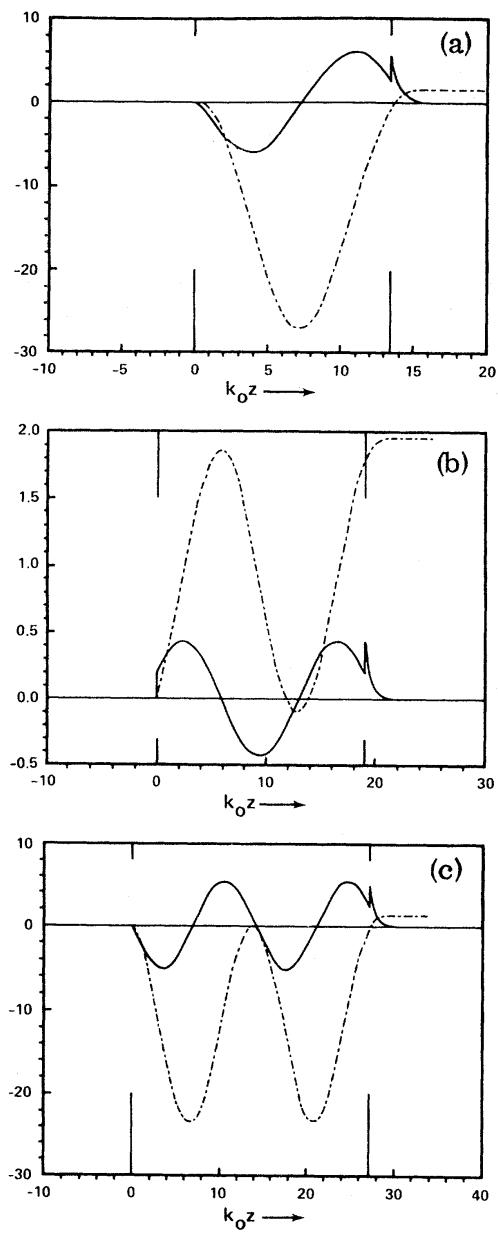

FIG. 4. The ponderomotive force (in units of $E_{0}{ }^{2} k_{0}$ / $8 \pi$ ) and the integral of the ponderomotive force for the same three cases as in Fig. 3. $F_{p},-\cdots-k_{0} \int F_{p} d z$,

different values of the first-step density. Again, the resonances become quite strong for correctly chosen layer thicknesses. Also, as with the planar case, the ponderomotive force at the outer edge of the first step is into the vacuum for the "on-resonance" cases, and into the plasma for the "off-resonance" case. It is important to note that the integrated ponderomotive force (also shown in Fig. 4) in all cases is into the plasma, as it must be, since the wave has been reflected.

Certain obvious questions need to be addressed. First of all, since the structure resonances have been obtained with sharp-boundary electron density profiles, is it possible for smooth real plasma boundaries to exhibit similar effects? Both the linear-ramp studies referred to earlier, which show increased electric field strength, and

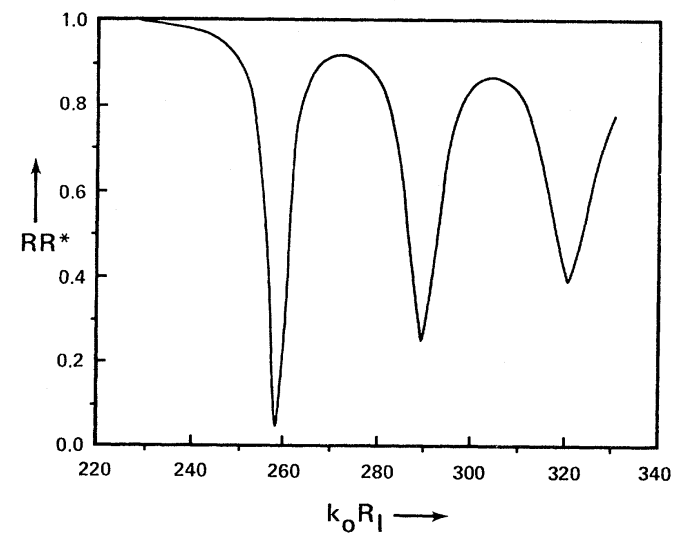

FIG. 5. Power reflectivity for the spherical two-step plasma layer for the following parameters: $n_{2} / n_{c}=1.1$; $\nu_{2} / \omega=0.001 ; \nu_{1} / \omega=0.001 ; n_{1} / n_{c}=0.99$.

our preliminary computer-code results for smooth boundary profiles suggest that the coherence effects will persist. These results will be the subject of a forthcoming paper.

Second, do coherence effects play a role in selfconsistent density-profile modifications? We believe they must because in the "on-resonance" cases the ponderomotive force may be many times larger than in the "off-resonance" cases so that if a resonance is established even for a short period, its effect may be strong. Finally, and perhaps most important, is it possible to establish "on-resonance" profiles either hydrodynamically, or structurally by pellet construction, to provide the calculated, strong absorption? Here we believe that considerably more study, both analytic and by hydro-codes, is required before these questions can be fully answered. The spherically symmetric hydrodynamic-electromagnetic wave interaction problem, wherein coherent plasma optics may play a role, is presently under investigation.

${ }^{1}$ R. B. White and F. F. Chen, Plasma Phys. 16,565 (1974); B. Bezzerides et al., in Proceedings of the Sixth International Conference on Plasma Physics and Controlled Nuclear Fusion Research, Berchtesgaden, West Germany, 1976 (International Atomic Energy Agency, Vienna, 1977), p. 123; E. Valeo et al., ibid., p. 133; D. W. Forslund et al., Phys. Rev. A 11, 679 (1975); K. G. Estabrook et al., Phys. Fluids 18,1152 (1975); J. M. Kindel et al., Phys. Rev. Lett. $\underline{34}, 134$ (1975). 
${ }^{2}$ Yu. M. Aliev et al., Phys. Rev. A 15, 2120 (1977).

${ }^{3}$ H. Hora, Phys. Fluids 12, $182(1969)$; J. Dawson et al., Phys. Fluids 12, 875 (1969). R. E. Kidder, in Proceedings of the Japan-U. S. Seminar on Laser Interaction with Matter, Kyoto, Japan, 1972, edited by K. Yamanaka (Japan Society for the Promotion of Science, Tokyo, 1973).

${ }^{4}$ N. G. Denisov, Zh. Eksp. Teor. Fiz. 31, 609 (1956) [Sov. Phys. JETP 4 , 544 (1971)]; V. L. Ginsberg, The Propagation of Electromagnetic Waves in Plasmas, (Addison-Wesley, Reading, Mass., 1969), pp. 193-228;
J. D. Lindl and P. K. Kaw, Phys. Fluids 14, 371 (1971); R. K. Osborn, IEEE Trans. Plasma Sci. $\underline{3}, 116$ (1975); White and Chen, Ref. 1.

${ }^{5}$ P. Mulser and C. van Kessel, Phys. Rev. Lett. $\underline{38}$, 902 (1977).

${ }^{6}$ K. A. Brueckner, and R. J. Janda, Nucl. Fusion 17 , 451 (1977); J. Virmont et al., to be published.

${ }^{7}$ K. Lee et al., Phys. Fluids 20, 51 (1977).

${ }^{8} \mathrm{~L}$. M. Brekhovskikh, Waves in Layered Media (Academic, New York, 1960).

${ }^{9}$ I. P. French et al., Can. J. Phys. 39, 1273 (1961).

\title{
High-Energy Ion Expansion in Laser-Plasma Interactions
}

\author{
R. Decoste \\ University of Maryland, College Park, Maryland 20742 \\ and \\ B. H. Ripin \\ Naval Research Laboratory, Washington, D. C. 20375
}

(Received 18 July 1977)

\begin{abstract}
Measurements of energetic-ion energy distributions produced from $\mathrm{CD}_{2}$ and $\mathrm{CH}_{2}$ targets are compared with a numerical model. The model describes the ambipolar expansion of hot electrons and two relatively cold ion species from an electron pressure gradient. For $\mathrm{CD}_{2}$ the plasma expansion is adequately represented by a single ion species, whereas for $\mathrm{CH}_{2}$ two-ion fluids are required to account for the energy and the relative behavior of the high-energy ion species.
\end{abstract}

Historically, the high-energy ions in a laserproduced plasma are defined as a small group of ions transporting a significant fraction of the $a b-$ sorbed laser energy. Most expansion models ${ }^{1,2}$ indicate that the energetic ions are the direct consequence of the presence of high-energy electrons. Most plasma simulations ${ }^{1,3}$ use a single ion species to model the ion expansion. Here we show that, although the hot-electron expansion can account for the energy content of the fast ions, a multi-ion-species description is usually required to reproduce the measured high-energy ion distributions. Ion energy distributions measured from $\mathrm{CD}_{2}$ targets, where both predominant species, $\mathrm{C}^{6+}$ and $\mathrm{D}^{+}$, have the same charge-tomass ratio, are adequately represented by a single-ion-species expansion. For $\mathrm{CH}_{2}$ targets, however, a two-ion fluid description is required to reproduce the qualitative features of the ion expansion.

Typical single-shot energy distributions of highenergy ions from $\mathrm{Nd}$-laser irradiation ( $75 \mathrm{psec}$, $\sim 10^{16} \mathrm{~W} / \mathrm{cm}^{2}$ with an $\mathrm{f} / 2$ lens) of $\mathrm{CD}_{2}$ and $\mathrm{CH}_{2}$ thick-slab targets are shown in Figs. 1 and 2, respectively. The gross variations between the charge-collector oscilloscope traces such as shown in Figs. 1(b) and 2(b) are mainly due to the different viewing angle between the target normal and the analyzer axis. For both cases the analyzer axis was at $35^{\circ}$ with respect to the laser axis but the target normals in Figs. 1 and 2 were $35^{\circ}$ and $22^{\circ}$, respectively, from the laser axis toward the analyzer. The high-energy-ion electrostatic analyzer ${ }^{4}$ used has twelve channels with $10 \%$ species and energy resolution. Since this analyzer cannot resolve $\mathrm{C}^{6+}$ from $\mathrm{D}^{+}$ions, the $\mathrm{C}^{6+}$ were removed by allowing them to charge exchange with a nitrogen background gas $\left(1.5 \times 10^{-4}\right.$ Torr $)$ in the target chamber before entering the ion analyzer. The lowered ionization stages, originally $\mathrm{C}^{6+}$, can then be differentiated from $\mathrm{D}^{+}$ions.

The ion energy distribution is finally reconstructed from the summation of the recombination products. For either $\mathrm{CH}_{2}$ or $\mathrm{CD}_{2}$ targets no ionization stages lower than $\mathrm{C}^{5+}$ and only a small fraction of $\mathrm{C}^{5+}$ appeared under good vacuum conditions $\left(8 \times 10^{-7}\right.$ Torr $)$.

The multipeak structures on the $\mathrm{H}^{+}$and $\mathrm{D}^{+}$energy distributions were always observed but were nonreproducible in detail. ${ }^{4,5}$ The main difference 